\title{
Unilateral basal ganglia hyper intensity in a patient with poorly controlled diabetes
}

\author{
G.L.A.S.N. Suranji, S.C. Weerasinghe, P.J.P Peiris
}

Neurology unit, Teaching Hospital Kurunegala, Sri Lanka.

\begin{abstract}
40 years old man with type two diabetes presented to the neurology department with one-day history of abnormal involuntary movements of left upper limb. These were more likely of choreiform movements than convulsive type movements. He was conscious, rational and oriented. Neurological examination was normal and the abnormal movements did not occur after the admission. His blood sugar control was poor and it was $400 \mathrm{mg} / \mathrm{dl}$ on admission. Rest of the blood investigations were normal. He had a Contrast tomography (CT) brain scan to evaluate the abnormal upper limb movements. This showed hyperdense putamen (Fig. 1).
\end{abstract}

\section{Discussion}

Basal ganglia abnormalities in hyperglycemia is reported in radiology literature. Nonketotic hyperglycemia has been associated with a characteristic appearance of unilateral lesions of the basal ganglia which are typically contralateral to the side of the patient's presenting symptoms. On non-contrast head CT scans, hyper dense putamen (Fig. 1) and/or caudate nucleus contralateral to the side of the patient's symptoms as in our patient's case. This is the most frequently described finding associated with nonketotic hyperglycemia.

The putamen is almost always involved with variable additional involvement of other basal ganglia structures, but isolated involvement of the globus pallidus or caudate nucleus was not reported in literature. The fig. 2 shows normal anatomy of the striatum in a MRI brain scan.

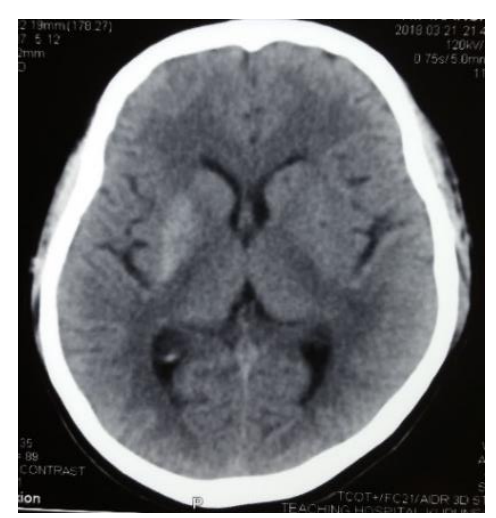

Figure 1: CT brain showing hyperdense putamen in nonketotic hyperglycemia.

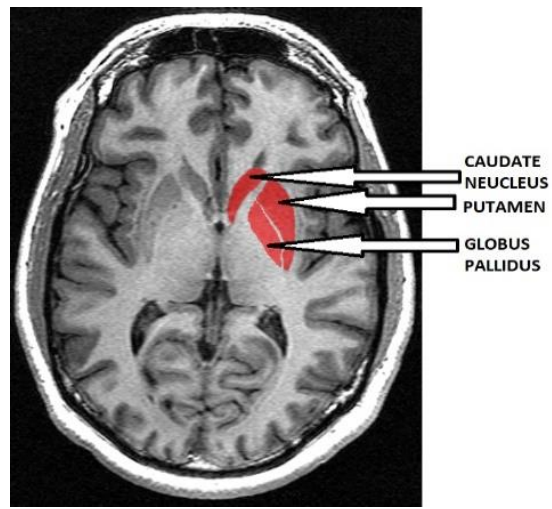

Figure 2: MRI brain scan showing normal anatomy of the striatum.

DOI: http://doi.org/10.4038/sjdem.v8i1.7353

Correspondence e-mail: sajithaw@yahoo.com

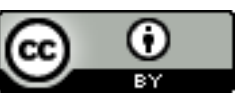

This is an open-access article distributed under the terms of the Creative Commons Attribution 4.0 International License, which permits unrestricted use, distribution and reproduction in any medium provided the original author and source are credited 
The cause for the hyperintense basal ganglia is not well understood. The most widely accepted hypothesis is hyperintensity of protein hydration layer in the cytoplasm of swollen gemistocytes (reactive astrocytes).

This hypothesis was supported by biopsies showing abundant gemistocytes in a hyperintense putaminal lesions. Alternate hypothesis includes putaminal petechial haemorrhage, demyelination like that seen in diabetic peripheral neuropathy, transient ischemic changes or desiccation seen as part of localized Wallerian degeneration.

Differential diagnoses for asymmetric basal ganglia hyperintensity on non-contrast head CT include haemorrhage and asymmetric calcification associated with underlying lesions such as developmental vascular anomalies. It should be kept in mind that nonketotic hyperglycemia can also present with bilateral hyperintense basal ganglia lesions.
Differential diagnosis for bilateral hyperintense basal ganglia is wide and includes; chronic liver disease (manganese toxicity), hypoxic-ischemic changes, disorders of calcium metabolism, Fahr disease, neurofibromatosis and Wilson disease.

Follow-up brain imaging for our patient is not available to assess the basal ganglia after normalization of serum glucose. However, the imaging findings in this case are typical of those reported for nonketotic hyperglycemic states.

This case highlights the importance of identifying the brain imaging features of hyperglycemia so unnecessary investigations and treatment could be avoided.

\section{References}

1. Barry G. Hansford, Dara Albert, Edward Yang. Classic neuroimaging findings of nonketotic hyperglycemia on computed tomography and magnetic resonance imaging with absence of typical movement disorder symptoms (hemichorea-hemiballism). J Radiol Case Rep. 2013 Aug; 7(8): 1-9. Published online 2013 Aug 1.

2. Nagai C, Kato T, Katagiri T, Sasaki H. Hyperintense putamen on T1-weighted MR images in a case of chorea with hyperglycemia. AJNR Am J Neuroradiol. 1995 Jun-Jul;16(6):1243-6.

3. Cherian A, Thomas B, Baheti NN, Chemmanam T, Kesavadas C. Concepts and controversies in nonketotic hyperglycemia-induced hemichorea: further evidence from susceptibility-weighted MR imaging. J Magn Reson Imaging. 2009; 29:699-703. 